\title{
Role of tumor-associated macrophages in the angiogenesis of well-differentiated hepatocellular carcinoma: Pathological-radiological correlation
}

\author{
NOBUHIRO FUJTAA ${ }^{1,2}$, AKIHIRO NISHIE ${ }^{1}$, SHINICHI AISHIMA ${ }^{2}$, YUICHIRO KUBO ${ }^{2}$, \\ YOSHIKI ASAYAMA ${ }^{1}$, KOUSEI ISHIGAMI ${ }^{1}$, DAISUKE KAKIHARA ${ }^{3}$, YASUHIRO USHIJIMA ${ }^{1}$, \\ YUKIHISA TAKAYAMA ${ }^{3}$, KEN SHIRABE $^{4}$, YOSHINAO ODA ${ }^{2}$ and HIROSHI HONDA ${ }^{1}$ \\ Departments of ${ }^{1}$ Clinical Radiology, ${ }^{2}$ Anatomic Pathology, ${ }^{3}$ Molecular Imaging and Diagnosis, and ${ }^{4}$ Surgery and Science, \\ Graduate School of Medical Sciences, Kyushu University, Fukuoka 812-8582, Japan
}

Received February 5, 2014; Accepted March 4, 2014

DOI: $10.3892 / o r .2014 .3138$

\begin{abstract}
The role of tumor-associated macrophages (TAMs) in hepatocellular carcinoma (HCC) has not been fully investigated. The aim of the present study was to clarify whether TAMs are associated with the angiogenesis of HCC during its multistep development, especially at an early stage. Forty-three well-differentiated HCCs and 30 well- to moderately differentiated HCCs (nodule-in-nodule lesion) were used. We immunohistochemically assessed microvessel density (by CD34) and macrophage count (by CD68 or CD163). Computed tomography hepatic angiography (CTHA) was performed for 26 well-differentiated HCCs and all 30 well- to moderately differentiated HCCs. The pathological analysis of the 43 welldifferentiated HCCs revealed a positive correlation between microvessel density and macrophage count $(\mathrm{P}=0.0026$, $\mathrm{r}=0.4486$ ). Based on the CTHA findings, 26 well-differentiated HCCs classified into a hyperattenuation group $(n=14)$ and a hypo- or isoattenuation group $(n=12)$. The microvessel density and macrophage count of the hyperattenuation group were significantly higher than those of the hypo- or isoattenuation group $(\mathrm{P}=0.0372$ and $\mathrm{P}=0.0476$ ). In the 30 well- to moderately differentiated HCCs, microvessel density of the moderately differentiated components was significantly higher than that of the well-differentiated components $(\mathrm{P}<0.0001)$. However, the macrophage count of the moderately differentiated component was significantly lower than that of the well-differentiated component $(\mathrm{P}<0.0001)$. All the moderately differentiated components showed marked hyperattenuation on CTHA.
\end{abstract}

Correspondence to: Dr Akihiro Nishie, Department of Clinical Radiology, Graduate School of Medical Sciences, Kyushu University, 3-1-1 Maidashi, Higashi-ku, Fukuoka 812-8582, Japan

E-mail: anishie@radiol.med.kyushu-u.ac.jp

Key words: hepatocellular carcinoma, tumor angiogenesis, tumorassociated macrophage, M2 macrophage, computed tomography hepatic angiography
Tumor vascularity was correlated with macrophage count in the tumor when limited to well-differentiated HCCs. TAMs may have a role in promoting angiogenesis of HCC at an early stage during its multistep development.

\section{Introduction}

Hepatocellular carcinoma (HCC) is one of the common malignancies in the world, and it is especially common in east Asia and sub-Saharan Africa (1-3). It is well known that the development of most HCCs is a multistep process under the presence of various chronic liver diseases $(4,5)$. During such hepatocellular carcinogenesis, changes in the blood supply have been reported, based on pathological and radiological studies (6-9). Briefly, a dysplastic nodule shows deterioration of arterial and portal blood flow, and in well-differentiated HCC (wHCC) arterial blood flow becomes dominant as a result of arterial neo-vascularization. Subsequently, classical (moderately and poorly differentiated) HCC shows abundant arterial blood flow and decreased portal blood flow.

The detection of wHCC is one of the most important issues in the management of patients with chronic liver disease. Previous studies have reported the imaging features of wHCCs, especially on computed tomography hepatic angiography (CTHA) and CT during arterial portography $(8,9)$. In those studies, wHCCs showed varying attenuation levels (hypoattenuation to hyperattenuation) on CTHA, suggesting that wHCC is the transitional stage of the arterial blood supply from a dysplastic nodule to classical HCC. However, to the best of our knowledge, the upregulation mechanism of angiogenesis in wHCC has not been fully elucidated.

Immunological studies have identified two distinct states of macrophages $(10,11)$. Classically activated (M1) macrophages activate microbial killing and show antitumor immunity. Tumor-associated macrophages (TAMs) are known to possess the immunosuppressive M2 macrophage phenotype. M2 macrophages are alternatively activated macrophages and contribute to tumor progression, including angiogenesis $(12,13)$. A significant correlation between TAMs and tumor vascularity has been reported in many tumors, including 
breast carcinoma (14), esophageal carcinoma (15), bladder (16) and prostate cancer (17).

However, it is known that macrophages in an HCC decrease as the histological grade advances, as confirmed by superparamagnetic iron oxide (SPIO)-enhanced magnetic resonance (MR) imaging findings (18). In fact, the macrophage count in wHCC is higher than that in classical (moderately and poorly differentiated) HCC (18). Considering these reports, TAMs may have a role in promoting angiogenesis of wHCCs.

In the present study, we attempted to clarify whether TAMs are associated with the angiogenesis of HCC, with a special focus on the early stage of HCC during the multistep development.

\section{Materials and methods}

Patients. The present study conformed to the ethical guidelines of the 1975 Declaration of Helsinki as revised in Tokyo 2004 and was performed with the approval of the Institutional Ethics Committee of Kyushu University. We could not obtain written informed consent from all the patients, therefore we removed identifying information from all samples before analysis for strict privacy protection. This procedure was in accordance with the Ethical Guidelines for Human Genome/ Gene Research enacted by the Japanese Government.

The cases included 73 HCCs surgically resected at our institution between March 1993 and September 2010. These consisted of 43 wHCCs and 30 well- to moderately differentiated HCCs (wmHCCs). Histological diagnosis was evaluated based on the classification proposed by the World Health Organization (19) and the International Consensus Group for Hepatocellular Neoplasia (20). WmHCC was defined as a nodule-in-nodule lesion when an inner nodule (moderately differentiated component) and an outer part (well-differentiated component) could be clearly separated. Patient details and tumor profiles are summarized in Table I. Twenty-six patients with wHCC and all 30 patients with wmHCCs underwent CTHA before surgery. Any cases with preceding therapy before surgery were excluded from the present study.

Immunohistochemistry. Ten-percent formalin-fixed, paraffinembedded tissue sections $(4 \mu \mathrm{m})$ containing sufficient tumor tissue were used for the study. Immunohistochemical staining was performed by the streptavidin-biotin-peroxidase method (Histofine SAB-PO kit; Nichirei, Tokyo, Japan). To retrieve antigens, the specimens were preincubated in trypsin (SigmaAldrich, St. Louis, MO, USA) in phosphate-buffered saline (PBS) for $30 \mathrm{~min}$ at $37^{\circ} \mathrm{C}$ for anti-CD34 antibody, while specimens were pretreated by heating in a microwave oven for $20 \mathrm{~min}$ for anti-CD68 and anti-CD163 antibody. The antibodies used in the present study were mouse monoclonal antibodies, and their dilutions were 1:50 for CD34 (QBEnd/10; Novocastra, Newcastle, UK), 1:300 for CD68 (KP-1; Dako, Glostrup, Denmark) and 1:200 for CD163 (10D6; Novocastra).

Evaluation of immunostaining. Microvessel density (MVD) was quantified using immunohistochemical staining for CD34 as a marker for endothelium following the method of a previous report (21). Three of the most vascularized areas were selected by scanning under a low-power view (x40), and the number of
Table I. Patient details and tumor profiles.

Characteristics

Data

\section{wHCC}

Gender (male/female)

Age (years) mean (range)

Hepatitis viral infection $(\mathrm{B} / \mathrm{C})$

$63.2(28-87)$

Liver cirrhosis

Tumor size $(\mathrm{cm})$ mean (range)

$1.6(0.6-3.8)$

wmHCC

Gender (male/female)

$16 / 14$

Age (years) mean (range)

$71.8(55-87)$

Hepatitis viral infection $(\mathrm{B} / \mathrm{C})$

Liver cirrhosis

$5 / 22$

Tumor size $(\mathrm{cm})$ mean (range)

$2.1(0.9-4.5)$

Data of gender, hepatitis virus infection and liver cirrhosis are numbers of patients. wHCC, well-differentiated hepatocellular carcinoma; wmHCC, well- to moderately differentiated hepatocellular carcinoma.

CD34-positive vessels was counted at a magnification of x100. The mean count of the three areas was defined as the MVD.

CD68-positive macrophages were also counted in both the tumor and the non-tumorous tissue. Five areas with the greatest number of macrophages were selected under a low-power view (x40), and the number of CD68-positive macrophages was counted at $\times 200$ magnification. The mean count of the five areas was defined as the macrophage count (MC). Macrophages in the portal tracts of the non-tumorous tissue were excluded from evaluation, as inflammatory cell infiltration in this region depends on the presence or condition of chronic hepatitis. To evaluate the MC in the tumor compared with the non-tumorous tissue, we defined the $\mathrm{MC}$ ratio as follows: $\mathrm{MC}$ ratio $=\mathrm{MC}$ in the tumor/ $\mathrm{MC}$ in the non-tumorous tissue. We also counted CD163-positive MC as a marker of M2 macrophages (22-24) in the same way we counted MC.

All measurements were performed by two pathologists (S.A. and Y.K.) without any knowledge of the clinicopathological findings.

CT protocols. CTHA was performed using a 4-row multidetector CT system (MDCT; Aquilion; Toshiba, Tokyo, Japan) or 16-row multidetector CT system (Aquilion). The scanning parameters of the 4-MDCT were as follows: collimation $3 \mathrm{~mm}$; pitch 5.5; reconstruction $5 \mathrm{~mm}$. The parameters of the 16-MDCT were: collimation $1 \mathrm{~mm}$; pitch 15 ; reconstruction $3 \mathrm{~mm}$. The hepatic arterial catheter for CTHA was placed in the proper, right or left hepatic arteries. Data acquisition of CTHA began $15 \mathrm{sec}$ (first phase) and $30 \mathrm{sec}$ (second phase) after the initiation of $15-40 \mathrm{ml}$ iopamidol solution $(150 \mathrm{mg} / \mathrm{ml})$ (Iopamiron 150; Bayer Health Care) at a rate of 1-2.5 ml/sec using an automated power injector. The appropriate injection rate for CTHA was determined as the maximum injection rate that would not cause a backward flow of contrast material on hepatic arteriography. The duration of the arterial injection was $15-20 \mathrm{sec}$. 

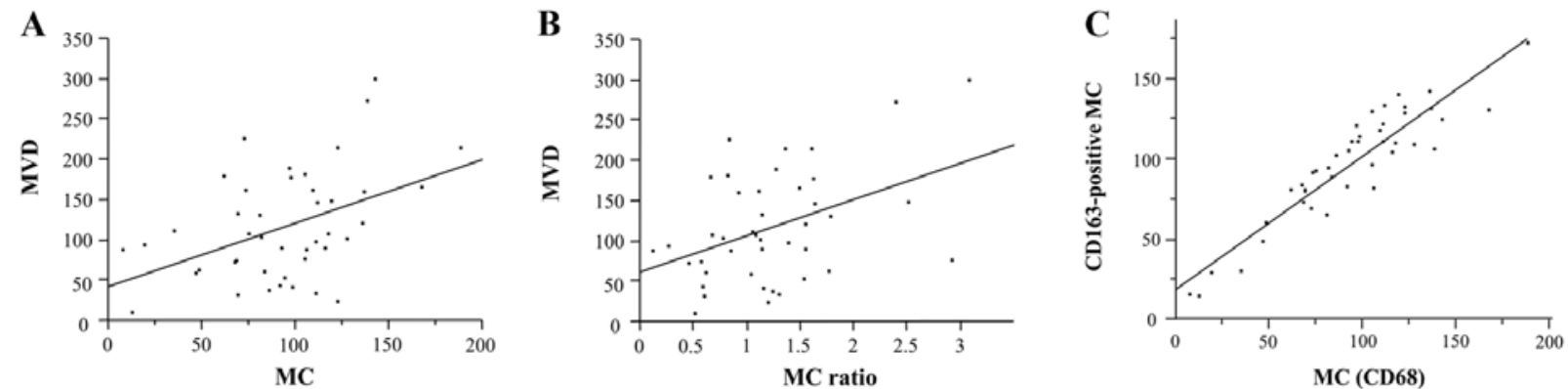

Figure 1. Correlation between microvessel density (MVD) and macrophage count (MC) or the MC ratio, and between MC and CD163-positive MC in welldifferentiated hepatocellular carcinomas. (A) A significant correlation was found between MVD and MC $(\mathrm{P}=0.0026, \mathrm{r}=0.4486)$. (B) A significant correlation was found between MVD and the MC ratio ( $\mathrm{P}=0.0039, \mathrm{r}=0.4308)$. (C) A strong correlation was found between $\mathrm{MC}$ and $\mathrm{CD} 163$-positive $\mathrm{MC}(\mathrm{P}<0.0001$, $\mathrm{r}=0.9173$ ). MC and CD163-positive MC showed an excellent agreement with an intraclass correlation coefficient of 0.913 .
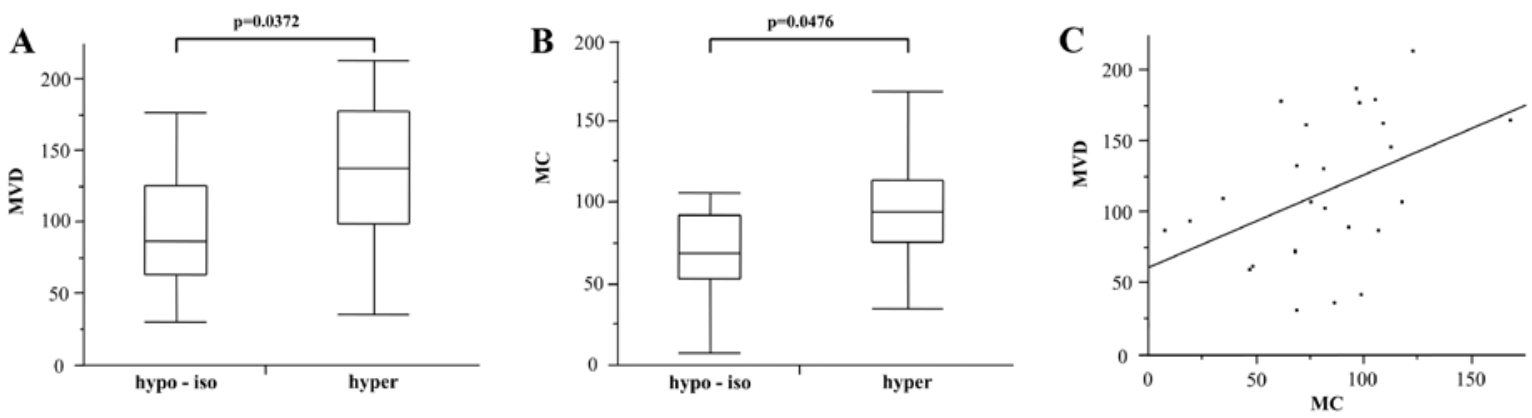

Figure 2. Correlation between the tumor attenuation level on the first phase of computed tomography hepatic angiography (CTHA), microvessel density (MVD) and macrophage count (MC) in well-differentiated hepatocellular carcinomas (wHCCs). (A) The MVD of the hyperattenuation group ( $\mathrm{n}=14$ ) was significantly higher than that of the hypo- or isoattenuation group $(\mathrm{n}=12, \mathrm{P}=0.0372)$. (B) The $\mathrm{MC}$ of the hyperattenuation group was significantly higher than that of hypo- or isoattenuation group ( $\mathrm{P}=0.0476)$. (C) A significant correlation was obtained between MVD and MC ( $\mathrm{P}=0.0270, \mathrm{r}=0.4334)$.

Imaging assessment. The CTHA images of HCCs were evaluated by two abdominal radiologists (N.F. and A.N.) in a consensus fashion. The observers were blinded to the pathological and clinical findings for each case. We evaluated the tumor attenuation level on the first phase of CTHA as we surmised that this phase represents tumor vascularity. On the basis of the enhancement pattern in relation to nontumorous liver, we divided the wHCCs into two groups: a hyperattenuation group and a hypo- or isoattenuation group. The hyperattenuation was determined when the whole lesion showed hyperattenuation or a hyperattenuation component was observed within the lesion on CTHA. Although the CTHA images of the wmHCCs were analyzed similarly, the two components were evaluated independently. In particular, we focused on the difference in the attenuation level on CTHA between the inner nodule (moderately differentiated component) and the outer part (well-differentiated component).

Statistical analysis. The relationships between the MVD and MC or MC ratio, and between MC and CD163-positive MC in the wHCCs were evaluated using the Pearson correlation test. Intraclass correlation coefficient was used to evaluate the agreement between MC and CD163-positive MC in the wHCCs. The correlation between the attenuation level on CTHA and the MVD or MC in wHCCs was analyzed by the Mann-Whitney U-test. In the wmHCCs, the MannWhitney U-test was used for comparison of the MVD or MC between the well- and moderately differentiated components. Continuous variables are expressed as means \pm SD. JMP 7.0.1 software (SAS Institute, Inc., Cary, NC, USA) and SPSS software version 13.0 (SPSS Inc., Chicago, IL, USA) were used for the analysis. P-values $<0.05$ were considered to indicate a statistically significant difference.

\section{Results}

Pathological and imaging analysis of the wHCCs. The pathological analysis of the $43 \mathrm{wHCCs}$ revealed an MVD of $116.8 \pm 67.6$ and an $\mathrm{MC}$ of $93.5 \pm 38.2$. The $\mathrm{MC}$ ratio was $1.2 \pm 0.6$. CD163-positive MC was $96.8 \pm 34.5$. A significant correlation was again obtained between MVD and MC $(\mathrm{P}=0.0026$, $\mathrm{r}=0.4486$; Fig. $1 \mathrm{~A})$ as well as between MVD and the MC ratio $(\mathrm{P}=0.0039, \mathrm{r}=0.4308$; Fig. 1B). In addition, a significant correlation was obtained between MC and CD163-positive MC $(\mathrm{P}<0.0001, \mathrm{r}=0.9173$; Fig. $1 \mathrm{C})$, and $\mathrm{MC}$ and $\mathrm{CD} 163$-positive MC showed an excellent agreement with an intraclass correlation coefficient of 0.913 .

Of the 26 wHCCs evaluated by CTHA, 12 wHCCs showed hypoattenuation $(\mathrm{n}=11)$ or isoattenuation $(\mathrm{n}=1)$ and $14 \mathrm{wHCCs}$ contained a hyperattenuation area on the first phase. The MVD and $\mathrm{MC}$ of these lesions were $115.2 \pm 51.8$ and $81.3 \pm 34.3$, respectively. The MVD of the hyperattenuation group $(132.4 \pm 52.4)$ was significantly higher than that of the hypo- or isoattenuation group (95.1 $\pm 45.0 ; \mathrm{P}=0.0372 ; \mathrm{Fig}$. $2 \mathrm{~A})$. In addition, the MC 

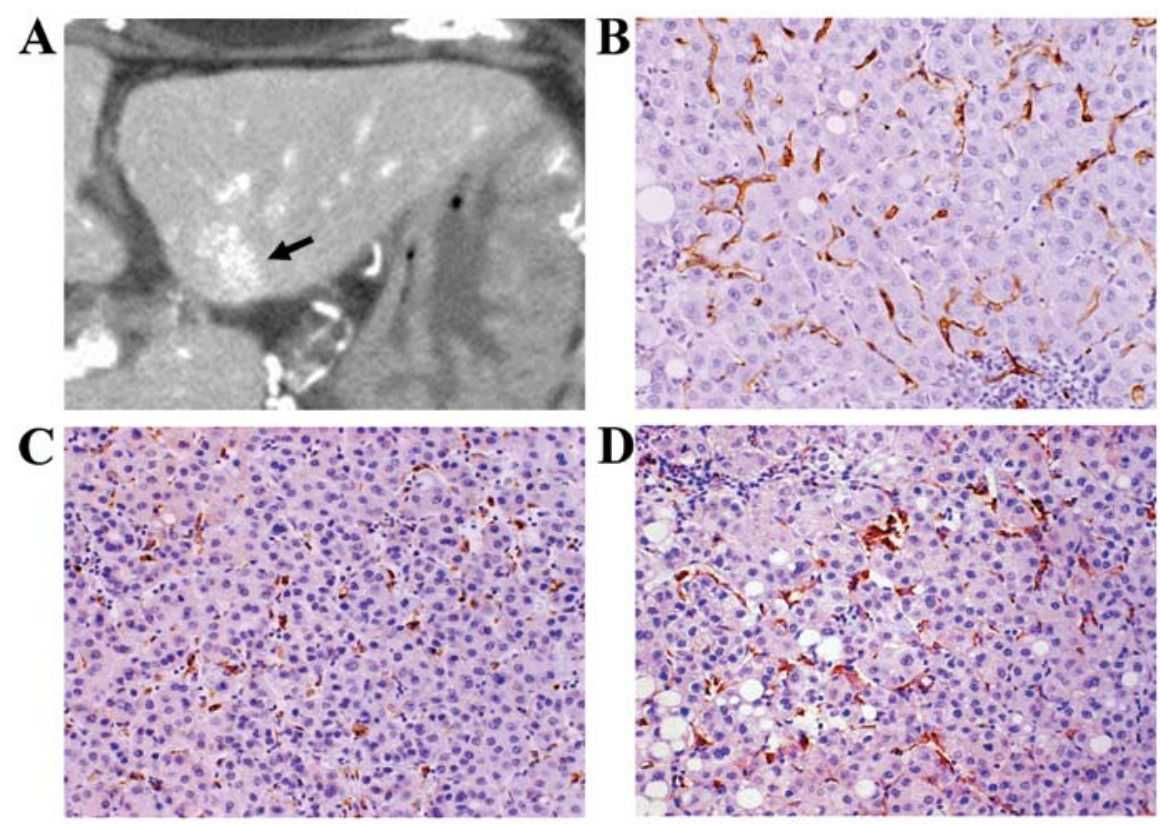

Figure 3. An 87-year-old woman with well-differentiated hepatocellular carcinoma in the left lobe of the liver. (A) CTHA image showed a hyperattenuating mass (arrow). (B) Immunohistochemical staining of CD34. A number of sinusoid-like vessels were observed in the tumor (MVD=178). Immunohistochemical staining of (C) CD68 and (D) CD163. A large number of CD68- or CD163-positive macrophages were observed in the tumor $(\mathrm{MC}=62, \mathrm{CD} 163$-positive $\mathrm{MC}=60)$.
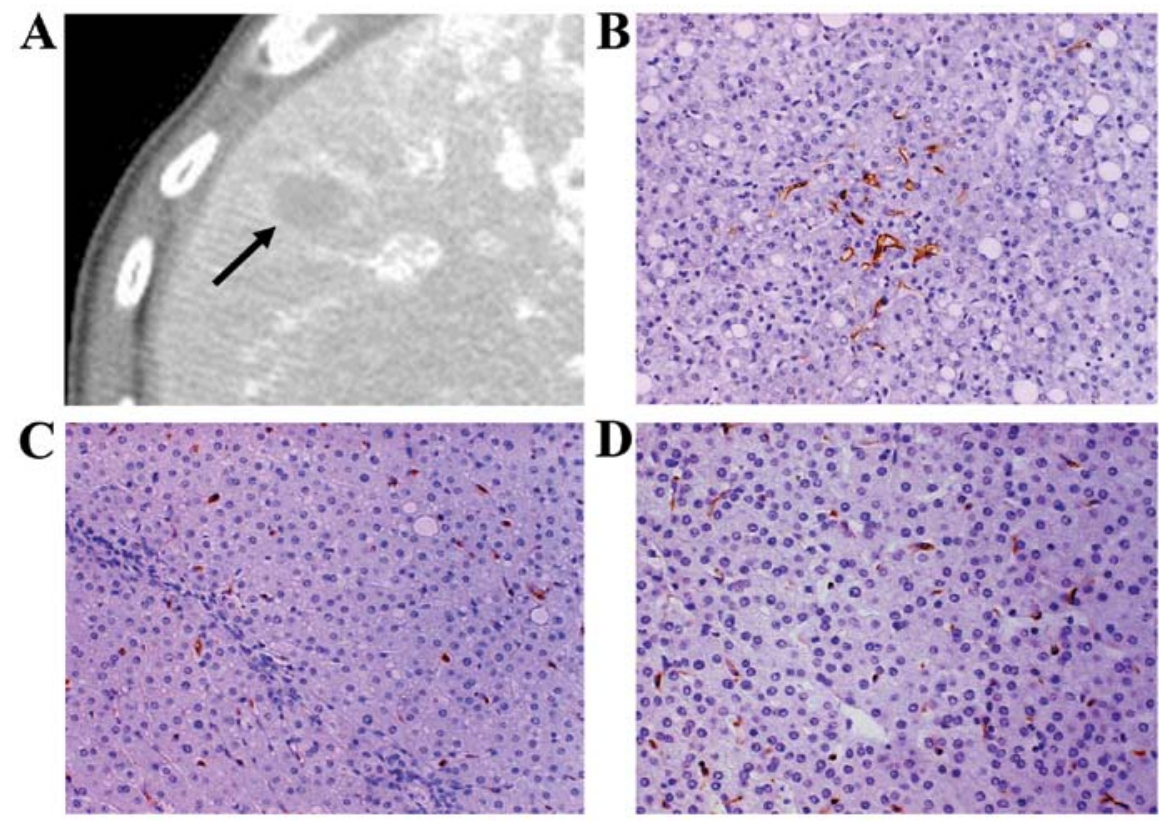

Figure 4. A 69-year-old man with well-differentiated hepatocellular carcinoma in the right lobe of the liver. (A) CTHA image showed a hypoattenuating mass (arrow). (B) Immunohistochemical staining of CD34. Small numbers of sinusoid-like vessels were observed in the tumor (MVD=41). Immunohistochemical staining of (C) CD68 and (D) CD163. CD68- or CD163-positive macrophages were scattered in the tumor (MC=19, CD163-positive MC=17).

of the hyperattenuation group $(92.8 \pm 36.0)$ was significantly higher than that of the hypo- or isoattenuation group $(67.8 \pm 27.8$; $\mathrm{P}=0.0476$; Fig. 2B). In these lesions, a significant correlation was obtained between MVD and MC $(\mathrm{P}=0.0270, \mathrm{r}=0.4334$; Fig. 2C). Two typical cases are shown in Figs. 3 and 4.

Pathological and imaging analysis of the wmHCCs. The MVD of the moderately differentiated components $(223.1 \pm 77.6)$ was significantly higher than that of the well-differentiated compo- nents $(65.9 \pm 35.9$; $\mathrm{P}<.0001$; Fig. $5 \mathrm{~A})$. In contrast, the $\mathrm{MC}$ of the moderately differentiated components $(76.8 \pm 29.5)$ was significantly lower than that of the well-differentiated components (127.6 $\pm 42.6, \mathrm{P}<.0001$; Fig. 5B). On the first phase of CTHA, all the moderately differentiated components showed marked hyperattenuation. Regarding the outer well-differentiated components, 12 lesions showed hypo- $(n=10)$ or isoattenuation $(n=2)$ and 18 lesions showed hyperattenuation. A representative case is shown in Fig. 6. 
A

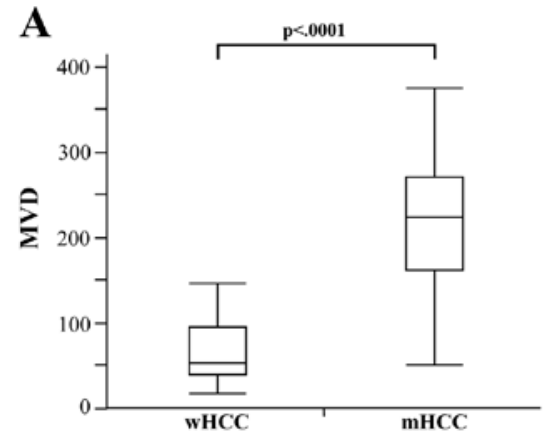

B

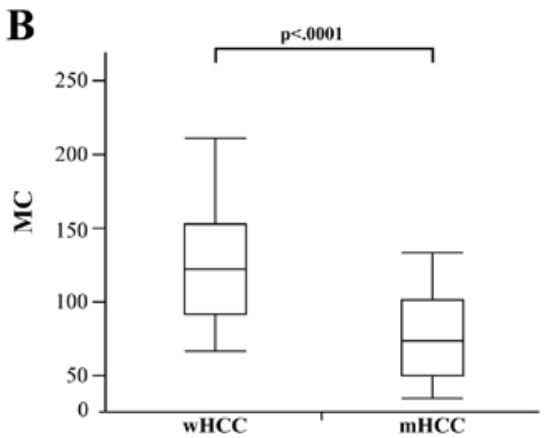

Figure 5. Correlation between histological grading, microvessel density (MVD) and macrophage count (MC) in well- to moderately differentiated hepatocellular carcinomas (nodule-in-nodule lesion). (A) The MVD of the moderately differentiated components was significantly higher than that of the well-differentiated components $(\mathrm{P}<0.0001)$. (B) The MC of the moderately differentiated components was significantly lower than that of the well-differentiated components $(\mathrm{P}<0.0001)$. wHCC, well-differentiated HCC; mHCC, moderately differentiated HCC.
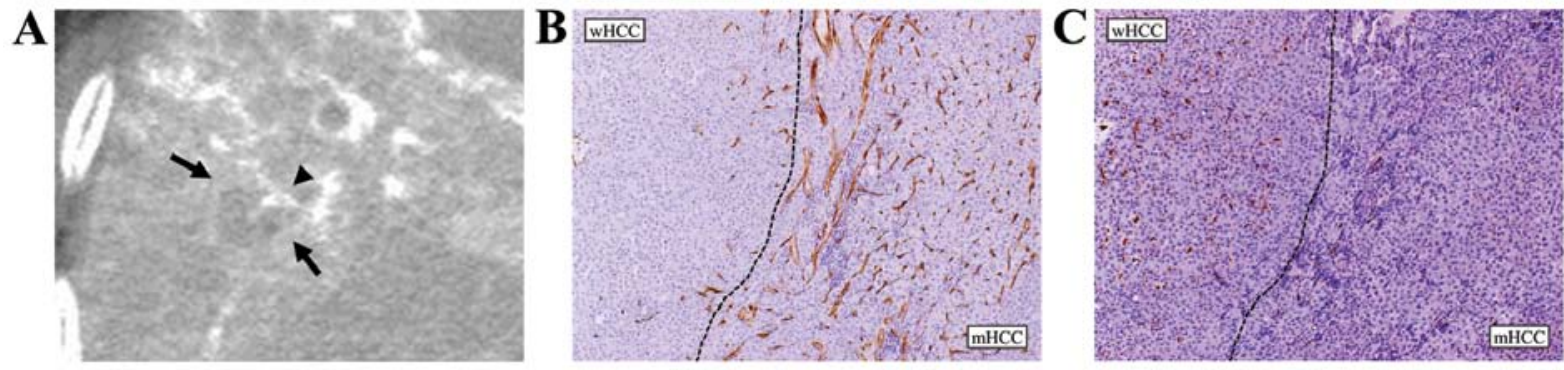

Figure 6. A 70-year-old woman with well- to moderately differentiated hepatocellular carcinoma in the right lobe of the liver. (A) CTHA image showed a mostly hypoattenuating mass (arrow) containing a hyperattenuating component (arrowhead). (B) Immunohistochemical staining of CD34. Small numbers of CD34-positive sinusoid-like vessels were observed in the well-differentiated component (MVD=51) and a large number of vessels were observed in the moderately differentiated component (MVD=156). (C) Immunohistochemical staining of CD68. A large number of CD68-positive macrophages was observed in the well-differentiated component $(\mathrm{MC}=155)$, and scattered macrophages were observed in the moderately differentiated component $(\mathrm{MC}=40)$. wHCC, well-differentiated HCC; mHCC, moderately differentiated HCC.

\section{Discussion}

The molecular mechanism of tumor angiogenesis has been well described $(25,26)$. Vascular endothelial growth factor (VEGF) and the angiopoietin (Ang) family are vascular endotheliumspecific growth factors (25). In HCCs, the correlation between such growth factors and MVD has been revealed in several studies (27-29). In addition, another study demonstrated that the stromal cells in solid tumors play an important role in tumor progression (30).

TAMs are particularly important components of the tumor microenvironment $(12,13)$. Macrophages are released from bone marrow, then circulate in the blood stream and migrate into tissues such as Kupffer cells in the liver. These macrophages are capable of lysing tumor cells, presenting antigens to $\mathrm{T}$ cells, and expressing immunostimulatory cytokines (12). In contrast, TAMs which are exposed to tumor-derived molecules such as interleukin (IL)-4 and IL-10 and are alternatively activated have poor antigen-presenting capability, and they produce factors that suppress T-cell proliferation and activity $(12,31)$. TAMs also release growth factors, cytokines and chemokines that regulate tumor growth, tumor invasion, and angiogenesis, such as VEGF (12). The role of TAMs in angiogenesis has been reported in many solid tumors (14-17).

In light of our present results, we hypothesize that TAMs play a crucial role in angiogenesis in wHCC, but not in moder- ately differentiated HCC. The mechanisms that control such changes of TAMs remain controversial. Stromal invasion, which means tumor cell invasion into the intratumoral portal tracts, is one of the most important pathologic features in wHCC (20). Stromal invasion decreases the number of portal veins and arteries, and may cause hypoxia. Murdoch et al (32) reported that TAMs accumulate in large numbers in avascular and hypoxia areas.

During the multistep development of wHCC, stromal invasion increases and causes increasing areas of hypoxia. We suspect that such hypoxic conditions recruit TAMs and promote angiogenesis in wHCC. In the present study, the wHCCs showed various attenuation levels on CTHA and various MVDs. The increasing stromal invasion during tumor development in wHCCs can explain the finding that the wHCCs showed such varying vascularity. However, in moderately differentiated HCC, VEGF and the Ang family that express in carcinoma cells play a central role in tumor angiogenesis, as previously described $(28,29)$. It no longer seems that TAMs play an important role in angiogenesis. A previous finding that the macrophage count in the wHCC is higher than that in the classical (moderately and poorly differentiated) HCC may also support our hypothesis (18). We assume that TAMs may induce an 'angiogenetic switch' at an early stage of HCC.

One issue that we should consider is whether macrophages in HCC are classical (M1) macrophages or immunosup- 
pressive M2 macrophages, as they both express CD68. We, therefore, compared the $\mathrm{MC}$ between the tumors and the nontumorous tissue. Twenty-eight of the 43 wHCCs $(65.1 \%)$ had higher MC in the tumor than in the non-tumorous tissue. In addition, a significant correlation was found between MVD and the MC ratio. We also compared the correlation between CD68- and CD163-positive macrophage counts. CD163 is a transmembrane scavenger receptor for haptoglobinhemoglobin complexes and is expressed in M2 macrophages (22-24). In the present study, a strong correlation was found between CD68- and CD163-positive macrophage counts. In addition, MC and CD163-positive MC showed an excellent agreement. Therefore, we consider that TAMs in HCC are M2 phenotype. Also, in previous studies, CD68-positive macrophages in the tumor were regarded as TAMs (14-16) which possess the immunosuppressive M2 macrophage phenotype.

Only a few radiological investigations have reported a correlation between imaging findings and these molecular mechanisms in tumor angiogenesis. Some reports described the relationship between imaging findings and VEGF expression in HCC $(33,34)$. Regarding TAMs, a role of macrophages in tumor progression in HCC under treatment with sorafenib, an oral multikinase inhibitor of VEGF, has been reported (35). Such molecular imaging studies will increase our understanding of the radiological imaging findings related to the molecular biologic environment and, in addition, should be used along with new target therapies for molecular mechanisms in tumor progression.

The present study has a few limitations. First, we divided the wHCCs into two groups (hyperattenuation group vs. isoor hypoattenuation group) in the evaluation of vascularity on CTHA as only one lesion showed isoattenuation. Second, our results cannot be compared with SPIO-enhanced MR imaging findings. Only two of our 43 patients with wHCC underwent SPIO-enhanced MR imaging. Since the uptake of SPIO in the tumor reflects the number and function of TAMs, we may be able to predict angiogenetic changes in tumors in the future using this method.

In conclusion, TAMs may have an important role in promoting angiogenesis in wHCCs, but not in moderately differentiated HCCs. Our findings suggest a role of TAMs in promoting angiogenesis at an early stage of HCC during its multistep development.

\section{Acknowledgements}

We thank Dr Yoshihiko Maehara, Department of Surgery and Science, Kyushu University, for providing the clinical information for this manuscript. The present study was supported by a Grant-in-Aid for Scientific Research (C) (24591814) from the Japanese Ministry of Education, Culture, Sports, Science, and Technology.

\section{References}

1. Okuda K: Hepatocellular carcinoma: clinicopathological aspects J Gastroenterol Hepatol 12: S314-S318, 1997.

2. Bosch FX, Ribes J, Díaz M and Cléries R: Primary liver cancer: worldwide incidence and trends. Gastroenterology 127: S5-S16, 2004.
3. Llovet JM, Burroughs A and Bruix J: Hepatocellular carcinoma. Lancet 362: 1907-1917, 2003.

4. Feitelson MA, Sun B, Satiroglu Tufan NL, Liu J, Pan J and Lian Z: Genetic mechanisms of hepatocarcinogenesis. Oncogene 21: 2593-2604, 2002.

5. Kudo M: Multistep human hepatocarcinogenesis: correlation of imaging with pathology. J Gastroenterol 44 (Suppl 19): 112-118, 2009.

6. Ueda K, Terada T, Nakanuma Y and Matsui O: Vascular supply in adenomatous hyperplasia of the liver and hepatocellular carcinoma: a morphometric study. Hum Pathol 23: 619-626, 1992.

7. Fujita N, Aishima S, Iguchi T, et al: Down-regulation of artery in moderately differentiated hepatocellular carcinoma related to tumor development. Hum Pathol 41: 838-847, 2010.

8. Tajima T, Honda H, Taguchi K, et al: Sequential hemodynamic change in hepatocellular carcinoma and dysplastic nodules: CT angiography and pathologic correlation. AJR Am J Roentgenol 178: 885-897, 2002.

9. Hayashi M, Matsui O, Ueda K, et al: Correlation between the blood supply and grade of malignancy of hepatocellular nodules associated with liver cirrhosis: evaluation by CT during intraarterial injection of contrast medium. AJR Am J Roentgenol 172: 969-976, 1999.

10. Biswas SK and Mantovani A: Macrophage plasticity and interaction with lymphocyte subsets: cancer as a paradigm. Nat Immunol 11: 889-896, 2010.

11. Martinez FO, Gordon S, Locati M and Mantovani A: Transcriptional profiling of the human monocyte-to-macrophage differentiation and polarization: new molecules and patterns of gene expression. J Immunol 177: 7303-7311, 2006.

12. Lewis CE and Pollard JW: Distinct role of macrophages in different tumor microenvironments. Cancer Res 66: 605-612, 2006.

13. Shirabe K, Mano Y, Muto J, et al: Role of tumor-associated macrophages in the progression of hepatocellular carcinoma. Surg Today 42: 1-7, 2012.

14. Leek RD, Lewis CE, Whitehouse R, Greenall M, Clarke J and Harris AL: Association of macrophage infiltration with angiogenesis and prognosis in invasive breast carcinoma. Cancer Res 56: 4625-4629, 1996.

15. Koide N, Nishio A, Sato T, Sugiyama A and Miyagawa S: Significance of macrophage chemoattractant protein-1 expression and macrophage infiltration in squamous cell carcinoma of the esophagus. Am J Gastroenterol 99: 1667-1674, 2004.

16. Hanada T, Nakagawa M, Emoto A, Nomura T, Nasu $N$ and Nomura Y: Prognostic value of tumor-associated macrophage count in human bladder cancer. Int J Urol 7: 263-269, 2000.

17. Lissbrant IF, Stattin P, Wikstrom P, Damber JE, Egevad L and Bergh A: Tumor associated macrophages in human prostate cancer: Relation to clinicopathological variables and survival. Int J Oncol 17: 445-451, 2000.

18. Imai Y, Murakami T, Yoshida S, et al: Superparamagnetic iron oxide-enhanced magnetic resonance images of hepatocellular carcinoma: correlation with histological grading. Hepatology 32: 205-212, 2000

19. Theise ND, Ishak KG, Kojiro M, et al: Hepatocellular carinoma. In: World Health Organization Classification of the Digestive System. Bosman FT, Carneiro F, Hruban RH and Theise ND (eds). IARC Press, Lyon, pp205-216, 2010.

20. International Consensus Group for Hepatocellular Neoplasia: Pathologic diagnosis of early hepatocellular carcinoma: a report of theInternational Consensus Group for Hepatocellular Neoplasia. Hepatology 49: 658-664, 2009.

21. Weidner N, Semple JP, Welch WR and Folkman J: Tumor angiogenesis and metastasis - correlation in invasive breast carcinoma. N Engl J Med 324: 1-8, 1991.

22. Hasita H, Komohara Y, Okabe H, et al: Significance of alternatively activated macrophages in patients with intrahepatic cholangiocarcinoma. Cancer Sci 101: 1913-1919, 2010.

23. Fujiwara Y, Komohara Y, Ikeda T and Takeya M: Corosolic acid inhibits glioblastoma cell proliferation by suppressing the activation of signal transducer and activator of transcription-3 and nuclear factor-kappa B in tumor cells and tumor-associated macrophages. Cancer Sci 102: 206-211, 2011.

24. Komohara Y, Ohnishi K, Kuratsu J and Takeya M: Possible involvement of the $\mathrm{M} 2$ anti-inflammatory macrophage phenotype in growth of human gliomas. J Pathol 216: 15-24, 2008.

25. Yancopoulos GD, Davis S, Gale NW, Rudge JS, Wiegand SJ and Holash J: Vascular-specific growth factors and blood vessel formation. Nature 407: 242-248, 2000. 
26. Holash J, Wiegand SJ and Yancopoulos GD: New model of tumor angiogenesis: dynamic balance between vessel regression and growth mediated by angiopoietins and VEGF. Oncogene 18: 5356-5362, 1999.

27. El-Assal ON, Yamanoi A, Soda Y, et al: Clinical significance of microvessel density and vascular endothelial growth factor expression in hepatocellular carcinoma and surrounding liver: possible involvement of vascular endothelial growth factor in the angiogenesis of cirrhotic liver. Hepatology 27: 1554-1562, 1998.

28. Wada H, Nagano H, Yamamoto H, et al: Expression pattern of angiogenic factors and prognosis after hepatic resection in hepatocellular carcinoma: importance of angiopoietin-2 and hypoxia-induced factor-1 $\alpha$. Liver Int 26: 414-423, 2006.

29. Mitsuhashi N, Shimizu H, Ohtsuka M, et al: Angiopoietins and Tie-2 expression in angiogenesis and proliferation of human hepatocellular carcinoma. Hepatology 37: 1105-1113, 2003.

30. de Visser KE, Eichten A and Coussens LM: Paradoxical roles of the immune system during cancer development. Nat Rev Cancer 6: $24-37,2006$
31. Mantovani A, Sozzani S, Locati M, Allavena P and Sica A: Macrophage polarization: tumor-associated macrophages as a paradigm for polarized M2 mononuclear phagocytes. Trends Immunol 23: 549-555, 2002.

32. Murdoch C, Giannoudis A and Lewis CE: Mechanisms regulating the recruitment of macrophages into hypoxic areas of tumors and other ischemic tissues. Blood 104: 2224-2234, 2004.

33. Kanematsu M, Osada S, Amaoka N, et al: Expression of vascular endothelial growth factor in hepatocellular carcinoma and the surrounding liver: correlation with angiographically assisted CT. AJR Am J Roentgenol 183: 1585-1593, 2004.

34. Kanematsu M, Osada S, Amaoka N, et al: Expression of vascular endothelial growth factor in hepatocellular carcinoma and the surrounding liver and correlation with MRI findings. AJR Am J Roentgenol 184: 832-841, 2005.

35. Zhang W, Zhu XD, Sun HC, et al: Depletion of tumor-associated macrophages enhances the effect of sorafenib in metastatic liver cancer models by antimetastatic and antiangiogenic effects. Clin Cancer Res 16: 3420-3430, 2010. 